\title{
Peripheral $\mathrm{N} \alpha$ scattering: a tool for identifying the two pion exchange component of the NN potential
}

\author{
L. A. Barreiro, R. Higa, C. L. Lima, and M. R. Robilotta \\ Nuclear Theory and Elementary Particle Phenomenology Group \\ Instituto de Física, Universidade de São Paulo, \\ Caixa Postal 66318, 05315-970, São Paulo, SP, Brazil
}

(November 5, 2018)

\begin{abstract}
We study elastic $\mathrm{N} \alpha$ scattering and produce a quantitative correlation between the range of the effective potential and the energy of the system. This allows the identification of the waves and energies for which the scattering may be said to be peripheral. We then show that the corresponding phase shifts are sensitive to the tail of the NN potential, which is due to the exchange of two pions. However, the present uncertainties in the experimental phase shifts prevent the use of $\mathrm{N} \alpha$ scattering to discriminate the existing models for the NN interaction.

PACS numbers: 21.30.-x, 13.75.Cs, 25.10.+s
\end{abstract}

\section{INTRODUCTION}

In the present understanding of the $N N$ interaction, long-range effects are ascribed to single pion exchanges, intermediate components are associated with exchanges of two and three pions, $\rho$ 's and $\omega$, as well as baryon excitations, such as the $\Delta$. At short distances, quark dynamics is supposed to dominate.

The long-range one-pion exchange potential (OPEP) became well established in the 1960's [1] as a necessary component of all realistic forces. This achievement was followed by an effort to determine the next layer of the interaction, associated with the two-pion exchange potential (TPEP), and various strategies were proposed to describe it. Early in 1971, Brown and Durso [2] pointed out that this component of the force is directly related to off-shell $\pi N$ scattering and hence strongly influenced by chiral symmetry. In a subsequent paper, Chemtob, Durso, and Riska [3] explored this relationship and investigated the phenomenological features of the TPEP obtained by means of dispersion relations. A very important step in this research program was the construction of the Paris potential [四, where dispersion relations were used to relate empirical information about the $\pi N$ process to the intermediate part of the force. This potential is rather successful in describing experimental data.

The intermediate part of the potential may also be studied in the framework of field theory. In this case, one writes down a Lagrangian involving the relevant degrees of freedom and then evaluate a certain number of Feynman diagrams. This leads to an amplitude which is afterwards transformed into a potential. An important early work along this line was that of Partovi and Lomon [5], who considered a Lagrangian containing just pions and nucleons with a pseudoscalar (PS) coupling and evaluated the box and crossed box diagrams contributing to the exchange of two uncorrelated pions. A detailed study of the same diagrams using a pseudovector (PV) coupling was performed later by Zuilhof and Tjon [6. The inclusion of the exchanges of resonances and baryon excitations led to the construction of the Bonn potential 7,8 , which is also quite successful in reproducing experimental data.

In a parallel line of development, several phenomenological potentials were constructed, which are also able to account for experimental information with the aid of adjusted parameters [9] 12].

An interesting feature of all approaches to the intermediate part of the potential is that their theoretical reliability decreases as one moves from the outer to the inner region. The intermediate range interactions exhibit a marked spatial hierarchy. The spatial features of a given interaction are determined by the mass exchanged in the $t$ channel. In the intermediate part of the potential, the lightest system that can be exchanged involves just two pions and has a mass of about $300 \mathrm{MeV}$. Other important effects, associated with resonances such as the $\rho$ and the $\omega$ are short ranged, because these states have masses around $750 \mathrm{MeV}$. When field theory is used, predictions for the inner parts of the mesonic sector of the potential are heavily influenced by form factors, bringing a lot of uncertainty to calculations. In the case of dispersion relations, on the other hand, predictions for the inner region are based on data which need to be extrapolated far away from their experimental region.

Recently there has been a renewal in the interest on the intermediate part of the $N N$ interaction, motivated by the realization that chiral symmetry provides a suitable theoretical framework for the calculation of a strong process 113 [16. In its minimal version, the chiral intermediate potential is based on a system containing just pions and nucleons. The theoretical foundations of this part of the potential are rather well established and it is reasonable to expect that it should become a standard ingredient of any modern $N N$ potential. A shortcoming of the minimal 
chiral potential is that it fails to reproduce experimental information in the case of the intermediate $\pi N$ amplitude entering in the TPEP. In order to overcome this difficulty, one may extend the chiral model, so as to encompass other degrees of freedom. This possibility was recently considered by Ordóñez, Ray, and van Kolck [17], who have shown that the inclusion of $\Delta$ 's in the model improves its predictive power. Alternatively, one may choose to introduce the empirical information that is missing in the intermediate $\pi N$ amplitude in a model independent way, with the help of the Höhler-Jacob-Strauss [18] subthreshold coefficients [19, 20]. This led to a TPEP which yields a satisfactory description of $N N$ data for waves and energies associated with peripheral scattering [21.22].

In spite of the considerable amount of activity related to the intermediate part of the $N N$ potential in the last twentyfive years, no consensus was reached about the fine details of this component of the force. The dynamical content of the various models is not uniform and the profile functions they yield for different components of the potential do not agree. This picture poses the problem of defining criteria for establishing the merits and shortcomings of the various existing potentials, so that a choice can be produced. An obvious criterion of choice is the ability a given potential has of reproducing experimental data. This possibility has been on for a long time and does not work as a distinguishing criterion, because all existing modern potentials are able to explain well experimental $N N$ phase shifts, but do so with the help of several free parameters that are adjusted ad hoc. Another problem about relying on $N N$ observables is that the OPEP contributes to all channels and waves, making it difficult to isolate unambiguously the contribution of intermediate range dynamics. It would therefore be interesting to find ways of obtaining information about the intermediate part of the potential directly from empirical data.

In this work we speculate about the possibility of obtaining such an information from the study of nucleon-alpha $(N \alpha)$ scattering. The $\alpha$ particle is a rather suitable system for the study of the intermediate part of the nuclear interaction, because it is a boson that has no spin and isospin and hence cannot couple to a single pion. This means that the outer part of the nuclear potential surrounding the $\alpha$ involves two uncorrelated pions. The scalar-isoscalar channel of the two-pion exchange $N N$ potential has strong central and spin-orbit components, and the same happens with the $N \alpha$ effective interaction. This picture has empirical support, since $N \alpha$ experimental phase shifts show that central and spin-orbit effects are very important [23,26,25,24]. Therefore we may expect that the peripheral scattering of nucleons by the $\alpha$ should be heavily dominated by two-pion exchange process and hence reflect the various approaches adopted in different $N N$ models. When low-energy protons are used as probes, the Coulomb barrier prevents short distance interactions. Thus the effects due to the nuclear force show themselves as deviations from Coulomb amplitudes, similarly to what happens in low-energy nucleus-nucleus collisions [27,28]. For instance, in sub-Coulomb $\mathrm{Pb}+\mathrm{Pb}$ Mott scattering the intermediate range $N N$ interaction accounts for deviations observed experimentally [27]. In the case of low-energy neutrons, on the other hand, angular momentum may be used to select the various regions of the potential.

This work is organized as follows. The effective $N \alpha$ potential is defined in sect. II and explicitly constructed in sect. [II]. In sect. IV we display our dynamical equations, which are based on the variable phase method. Finally, in sect. $\mathrm{V}$ we discuss our results.

\section{DYNAMICS}

In this section we present briefly the main equations used in this work. In a complete treatment of the $N \alpha$ scattering problem, one has to deal with a rather complex five-body system. However, here we are interested in estimating the effects of the tail of the $N N$ interaction, which manifests themselves at large distances and high values of the angular momentum. We therefore assume that the $\alpha$ subsystem remains undisturbed during the interaction.

The wave function for the four-body nuclear system ${ }^{4} \mathrm{He}$ can be written, in terms of the spatial, spin, and isospin degrees of freedom, as

$$
\begin{aligned}
\left|N_{1} \cdots N_{4}\right\rangle & \left.=\left|\mathbf{r}_{1} \cdots \mathbf{r}_{4}\right\rangle \otimes|\operatorname{spin}\rangle \otimes \mid \text { isospin }\right\rangle \\
& =|\mathbf{R}\rangle|\alpha\rangle
\end{aligned}
$$

where the collective variable $\mathbf{R}$ is given in terms of the individual coordinates $\mathbf{r}_{i}$ :

$$
\mathbf{R}=\frac{1}{4} \sum_{i=1}^{4} \mathbf{r}_{i}
$$

and $|\alpha\rangle$ represents the ${ }^{4} \mathrm{He}$ ground state. This wave function is known to have $T=0, J^{\pi}=0^{+}$, and both $S$ and $D$ spatial components [29]. In order to simplify the calculation we neglect $D$ waves, adopt a Gaussian structure for the spatial wave function [29] and write 


$$
|\alpha\rangle=N_{\alpha} \exp \left(-\frac{\alpha}{2} \sum_{i>j=1}^{4} \mathbf{r}_{i j}^{2}\right)\left|\chi_{\alpha}\right\rangle
$$

where $\mathbf{r}_{i j}=\left(\mathbf{r}_{i}-\mathbf{r}_{j}\right), N_{\alpha}$ is a normalization constant, $\alpha$ is a parameter extracted from Ref. [29], and $\left|\chi_{\alpha}\right\rangle$ is the spin-isospin wave function.

The dynamics of the four body system is determined by the Hamiltonian

$$
\begin{aligned}
H_{4} & =\sum_{i=1}^{4}-\frac{\nabla_{i}^{2}}{2 m}+\sum_{i>j=1}^{4} \Theta_{i j} \\
& =-\frac{\nabla_{\mathrm{R}}^{2}}{2 M_{\alpha}}+H_{\alpha},
\end{aligned}
$$

where $\Theta_{i j}=k \mathbf{r}_{i j}^{2} / 2$ is the harmonic potential and $k=4 \alpha^{2} / m$.

In order to isolate the motion of the center of mass, it is useful to use the Jacobi variables, given generically by

$$
\boldsymbol{\rho}_{i}=\sum_{j=1}^{4} a_{i j} \mathbf{r}_{j}
$$

where the coefficients $a_{i j}$ are summarized in the matrix

$$
\boldsymbol{a}=\left(\begin{array}{cccc}
\sqrt{1 / 2} & -\sqrt{1 / 2} & 0 & 0 \\
\sqrt{1 / 6} & \sqrt{1 / 6} & -\sqrt{2 / 3} & 0 \\
\sqrt{1 / 12} & \sqrt{1 / 12} & \sqrt{1 / 12} & -\sqrt{3 / 4}
\end{array}\right) .
$$

In terms of these new coordinates, the Hamiltonian for the isolated four-body system is

$$
H_{4}=-\frac{\nabla_{\mathrm{R}}^{2}}{2 M_{\alpha}}+H_{\alpha}
$$

where $-\nabla_{\mathrm{R}}^{2} / 2 M_{\alpha}$ describes the center-of-mass motion and

$$
H_{\alpha}=\sum_{i=1}^{3}\left[-\frac{\nabla_{\rho_{i}}^{2}}{2 m_{i}}+2 k \rho_{i}^{2}\right]
$$

is the intrinsic four-body Hamiltonian. The Schrödinger equation for the $\alpha$ particle is

$$
H_{\alpha}|\alpha\rangle=E_{\alpha}|\alpha\rangle,
$$

where $E_{\alpha}=M_{\alpha}-4 m$ and $|\alpha\rangle$ is now given by

$$
|\alpha\rangle=\left(\frac{4 \alpha}{\pi}\right)^{9 / 4} \exp \left(-2 \alpha \sum_{i=1}^{3} \boldsymbol{\rho}_{i}^{2}\right)\left|\chi_{\alpha}\right\rangle .
$$

The strong interaction between the incoming particle, hereafter labeled by $o$, and the nucleon $i$ within the $\alpha$ particle is denoted by $V_{o i}\left(\mathbf{r}_{o i}\right)$. The study of the effects of this interaction is the main object of this work.

The Schrödinger equation for this five nucleon system is

$$
H_{5}\left|N_{o} \cdots N_{4}\right\rangle=E\left|N_{o} \cdots N_{4}\right\rangle
$$

where

$$
\begin{aligned}
H_{5} & =\sum_{i=0}^{4}-\frac{\nabla_{i}^{2}}{2 m}+\sum_{i>j=1}^{4} \Theta_{i j}+\sum_{i=1}^{4} V_{o i} \\
& =H_{\alpha}-\frac{\nabla_{\mathrm{R}}^{2}}{2 M_{\alpha}}-\frac{\nabla_{o}^{2}}{2 m}+\sum_{i=1}^{4} V_{o i} .
\end{aligned}
$$


Approximating the five-body wave function by a product of the different clusters constituting the system

$$
\left|N_{o} \cdots N_{4}\right\rangle \simeq\left|N_{o}, \mathbf{R}\right\rangle|\alpha\rangle
$$

we can rewrite the dynamical equations as

$$
\begin{aligned}
H_{5}\left|N_{o} \cdots N_{4}\right\rangle & \simeq\left[E_{\alpha}-\frac{\nabla_{\mathrm{R}}^{2}}{2 M_{\alpha}}-\frac{\nabla_{o}^{2}}{2 m}+\sum_{i=1}^{4} V_{o i}\right]\left|N_{o}, \mathbf{R}\right\rangle|\alpha\rangle \\
& =E\left|N_{o}, \mathbf{R}\right\rangle|\alpha\rangle .
\end{aligned}
$$

Introducing two new Jacobi variables

$$
\begin{aligned}
& \mathbf{s}=\frac{1}{5}\left(\mathbf{r}_{o}+4 \mathbf{R}\right), \\
& \mathbf{x}=\mathbf{r}_{o}-\mathbf{R},
\end{aligned}
$$

we have

$$
\left[-\frac{\nabla_{\mathbf{s}}^{2}}{2\left(M_{\alpha}+m\right)}-\frac{\nabla_{\mathbf{x}}^{2}}{2 \mu}+\sum_{i=1}^{4} V_{o i}\right]\left|N_{o}, \mathbf{R}\right\rangle|\alpha\rangle=\left(E-E_{\alpha}\right)\left|N_{o}, \mathbf{R}\right\rangle|\alpha\rangle
$$

where

$$
\mu=\frac{M_{\alpha} m}{M_{\alpha}+m} \simeq \frac{4}{5} m
$$

Writing down explicitly the projectile wave function as

$$
\left|N_{o}\right\rangle=\left|\mathbf{r}_{o}\right\rangle\left|\chi_{o}\right\rangle
$$

and going to the C.M. frame of the five-body system, we obtain

$$
\left[-\frac{\nabla_{\mathbf{x}}^{2}}{2 \mu}+\sum_{i=1}^{4} V_{o i}\right]|\mathbf{x}\rangle\left|\chi_{o}\right\rangle|\alpha\rangle=E_{\mathrm{x}}|\mathbf{x}\rangle\left|\chi_{o}\right\rangle|\alpha\rangle,
$$

where $E_{\mathrm{x}}=E-E_{\alpha}$. Multiplying this equation by $\langle\alpha|$ and integrating over the $\alpha$ coordinates, we get

$$
\left[-\frac{\nabla_{\mathbf{x}}^{2}}{2 \mu}+W(\mathbf{x})\right]|\mathbf{x}\rangle\left|\chi_{o}\right\rangle=E_{x}|\mathbf{x}\rangle\left|\chi_{o}\right\rangle
$$

where

$$
W(\mathbf{x})=\left\langle\alpha\left|\sum_{i=1}^{4} V_{o i}\left(\mathbf{r}_{o i}\right)\right| \alpha\right\rangle
$$

is the effective $N-\alpha$ potential.

\section{THE EFFECTIVE POTENTIAL}

The two-body strong potential $V_{o i}$ is written as

$$
V_{o i}=\sum_{S, T} V_{o i}^{T S}\left(\mathbf{r}_{o i}\right) P_{o i}^{T} P_{o i}^{S}
$$

where the indices $T$ and $S$ represent the total isospin and spin of the $N N$ system whereas $P_{o i}^{T}$ and $P_{o i}^{S}$ are the corresponding projection operators. The explicit forms of the isospin operators are 


$$
\begin{aligned}
& P_{o i}^{0}=\frac{1}{4}\left[1-\boldsymbol{\tau}^{(o)} \cdot \boldsymbol{\tau}^{(i)}\right], \\
& P_{o i}^{1}=\frac{1}{4}\left[3+\boldsymbol{\tau}^{(o)} \cdot \boldsymbol{\tau}^{(i)}\right],
\end{aligned}
$$

and similar expressions hold for the spin degrees of freedom.

The various radial components $V_{o i}^{T S}$ have the following structure

$$
\begin{aligned}
V_{o i}^{T S}= & V_{C}^{T S}\left(\mathbf{r}_{o i}\right)+V_{L S}^{T S}\left(\mathbf{r}_{o i}\right) \mathbf{L}_{o i} \cdot\left(\frac{\boldsymbol{\sigma}^{(o)}}{2}+\frac{\boldsymbol{\sigma}^{(i)}}{2}\right) \\
& +V_{T}^{T S}\left(\mathbf{r}_{o i}\right)\left(3 \boldsymbol{\sigma}^{(o)} \cdot \hat{\mathbf{r}}_{o i} \boldsymbol{\sigma}^{(i)} \cdot \hat{\mathbf{r}}_{o i}-\boldsymbol{\sigma}^{(o)} \cdot \boldsymbol{\sigma}^{(i)}\right) \\
& + \text { small components. }
\end{aligned}
$$

In this expression, $\mathbf{L}_{o i}$ is the orbital angular momentum for the pair oi, whereas $V_{C}^{T S}, V_{L S}^{T S}$, and $V_{T}^{T S}$ represent the central, spin-orbit, and tensor components of the potential, respectively.

In the evaluation of the effective potential, one needs the expectation value of $V_{o i}$ between the wave functions of the $\alpha$ particle. Noting that $\left\langle\chi_{\alpha}\left|\boldsymbol{\sigma}^{(i)}\right| \chi_{\alpha}\right\rangle=\left\langle\chi_{\alpha}\left|\boldsymbol{\tau}^{(i)}\right| \chi_{\alpha}\right\rangle=0$, we have

$$
\left\langle\chi_{\alpha}\left|V_{o i}\left(\mathbf{r}_{o i}\right)\right| \chi_{\alpha}\right\rangle=V_{C}\left(\mathbf{r}_{o i}\right)+V_{L S}\left(\mathbf{r}_{o i}\right) \mathbf{L}_{o i} \cdot \frac{\boldsymbol{\sigma}^{(o)}}{2},
$$

where

$$
V_{C}\left(\mathbf{r}_{o i}\right)=\frac{1}{16}\left[V_{C}^{00}\left(\mathbf{r}_{o i}\right)+3 V_{C}^{10}\left(\mathbf{r}_{o i}\right)+3 V_{C}^{01}\left(\mathbf{r}_{o i}\right)+9 V_{C}^{11}\left(\mathbf{r}_{o i}\right)\right]
$$

and

$$
V_{L S}\left(\mathbf{r}_{o i}\right)=\frac{1}{4}\left[V_{L S}^{01}\left(\mathbf{r}_{o i}\right)+3 V_{L S}^{11}\left(\mathbf{r}_{o i}\right)\right] .
$$

The strong effective potential is then given by

$$
\begin{aligned}
W(\mathbf{x})= & \sum_{i=1}^{4}\left(\frac{4 \alpha}{\pi}\right)^{9 / 2} \int d \boldsymbol{\rho}_{1} d \boldsymbol{\rho}_{2} d \boldsymbol{\rho}_{3} \exp \left(-2 \alpha \sum_{j=1}^{3} \boldsymbol{\rho}_{j}^{2}\right) \\
& \times\left[V_{C}\left(\mathbf{r}_{o i}\right)+V_{L S}\left(\mathbf{r}_{o i}\right) \mathbf{L}_{o i} \cdot \frac{\boldsymbol{\sigma}^{(o)}}{2}\right] \exp \left(-2 \alpha \sum_{j=1}^{3} \boldsymbol{\rho}_{j}^{2}\right) .
\end{aligned}
$$

In the evaluation of this expression, it is convenient to note that

$$
\mathbf{r}_{i}=\mathbf{R}+\sum_{j=1}^{3} a_{i j}^{T} \boldsymbol{\rho}_{j}
$$

and hence

$$
\mathbf{r}_{o i}=\mathbf{x}-\sum_{j=1}^{3} a_{i j}^{T} \boldsymbol{\rho}_{j} .
$$

The angular momentum operator $\mathbf{L}_{o i}$ is given by $\mathbf{L}_{o i}=\mathbf{r}_{o i} \times \mathbf{p}_{o i}$, where $\mathbf{p}_{o i}=-i \nabla_{o i}$. The use of the chain rule allows us to write

$$
\begin{aligned}
\mathbf{L}_{o i}= & \left(\mathbf{x}-\sum_{j=1}^{3} a_{i j}^{T} \boldsymbol{\rho}_{j}\right) \times\left(\frac{1}{4} \mathbf{p}_{\mathbf{x}}-\sum_{k=1}^{3} a_{i k}^{T} \mathbf{p}_{k}\right) \\
= & \frac{1}{4} \mathbf{x} \times \mathbf{p}_{\mathbf{x}}-\frac{1}{4} \sum_{j=1}^{3} a_{i j}^{T}\left(\boldsymbol{\rho}_{j} \times \mathbf{p}_{\mathbf{x}}\right)-\sum_{k=1}^{3} a_{i k}^{T}\left(\mathbf{x} \times \mathbf{p}_{k}\right) \\
& +\sum_{k=1}^{3} \sum_{j=1}^{3} a_{i j}^{T} a_{i k}^{T}\left(\boldsymbol{\rho}_{j} \times \mathbf{p}_{k}\right) .
\end{aligned}
$$


When the operator $\mathbf{p}_{k}$ acts on the spatial wave function, we have

$$
\mathbf{p}_{k} \exp \left(-2 \alpha \sum_{i=1}^{3} \boldsymbol{\rho}_{i}^{2}\right)=-i(-4 \alpha) \boldsymbol{\rho}_{k} \exp \left(-2 \alpha \sum_{i=1}^{3} \boldsymbol{\rho}_{i}{ }^{2}\right) .
$$

Defining $\mathbf{L}_{\mathrm{x}}=\mathbf{x} \times \mathbf{p}_{\mathbf{x}}$, we write

$$
W(\mathbf{x})=W_{C}(\mathbf{x})+\frac{1}{4} W_{L S}(\mathbf{x}) \mathbf{L}_{\mathbf{x}} \cdot \frac{\boldsymbol{\sigma}^{(o)}}{2} .
$$

The Gaussian structure of the wave function allows several integrations to be performed analytically and we obtain

$$
W_{C}(\mathbf{x})=4\left(\frac{16 \alpha}{3 \pi}\right)^{1 / 2} \frac{1}{x} \int_{0}^{\infty} d u u V_{C}(u)\left\{\exp \left[-\frac{16 \alpha}{3}(x-u)^{2}\right]-\exp \left[-\frac{16 \alpha}{3}(x+u)^{2}\right]\right\}
$$

and

$$
\begin{aligned}
W_{L S}(\mathbf{x})= & 4\left(\frac{16 \alpha}{3 \pi}\right)^{1 / 2} \frac{1}{x^{2}} \int_{0}^{\infty} d u u V_{L S}(u) \times \\
& \left\{\left(u-\frac{3}{32 \alpha x}\right) \exp \left[-\frac{16 \alpha}{3}(x-u)^{2}\right]+\left(u+\frac{3}{32 \alpha x}\right) \exp \left[-\frac{16 \alpha}{3}(x+u)^{2}\right]\right\} .
\end{aligned}
$$

For a channel with total angular momentum $\mathbf{J}$ and orbital angular momentum $\mathbf{L}$, we obtain the following form for the effective potential:

$$
W_{J L}(x)=W_{C}(x)+\frac{1}{8}\left[J(J+1)-L(L+1)-\frac{3}{4}\right] W_{L S}(x) .
$$

Thus we have

$$
\begin{aligned}
& W_{L-\frac{1}{2} L}(x)=W_{C}(x)-\frac{1}{8}(L+1) W_{L S}(x), \\
& W_{L+\frac{1}{2} L}(x)=W_{C}(x)+\frac{1}{8} L W_{L S}(x) .
\end{aligned}
$$

\section{THE VARIABLE PHASE APPROACH}

We are interested in the properties of the tail of the $N N$ interaction and hence it is convenient to calculate the $N \alpha$ phase shifts by means of the so-called variable phase method, as described by Calogero [30]. This method is fully equivalent to the Schrödinger formalism, and has the advantage of providing a clear picture of the spatial influence of the potential over the phase shift and of its relationship with the centrifugal barrier. For the reader's convenience, we hereafter collect the main formulas needed in this calculation. As we disregard tensor interactions, there are only uncoupled channels. The wave $u_{J L}(r)$, representing a system with total and orbital angular momenta $J$ and $L$, respectively, is written in terms of the radial Green's functions as

$$
u_{J L}(r)=c_{J L}(k, r) \hat{j}_{L}(k r)-s_{J L}(k, r) \hat{n}_{L}(k r),
$$

where $k=\sqrt{2 \mu E_{x}}, \hat{j}_{L}(k r)$ and $\hat{n}_{L}(k r)$ are the usual spherical Bessel and Neumann functions multiplied by $k r$ whereas $c_{J L}(k, r)$ and $s_{J L}(k, r)$ are defined in terms of $V_{J L}(r)$ as follows:

$$
\begin{gathered}
c_{J L}(k, r)=1-\frac{1}{k} \int_{0}^{r} d r^{\prime} V_{J L}\left(r^{\prime}\right) \hat{j}_{L}\left(k r^{\prime}\right) u_{J L}\left(r^{\prime}\right), \\
s_{J L}(k, r)=-\frac{1}{k} \int_{0}^{r} d r^{\prime} V_{J L}\left(r^{\prime}\right) \hat{n}_{L}\left(k r^{\prime}\right) u_{J L}\left(r^{\prime}\right) .
\end{gathered}
$$

The phase shift $\delta_{J L}(k)$ is related to these functions by 


$$
\tan \delta_{J L}(k)=\lim _{r \rightarrow \infty} \frac{s_{J L}(k, r)}{c_{J L}(k, r)} .
$$

This motivates the definition of a variable phase $\delta_{J L}(k, r)$ through

$$
\delta_{J L}(k, r)=\arctan \left[\frac{s_{J L}(k, r)}{c_{J L}(k, r)}\right] .
$$

This function vanishes at the origin 30] and becomes the phase shift when $r$ approaches infinity. It is determined dynamically by means of a first-order differential equation, obtained by differentiating Eq. (4.5), using Eqs. (4.2), (4.3), and manipulating the result to obtain

$$
\frac{d}{d r} \delta_{J L}(k, r)=-\frac{1}{k} W_{J L}(r) P_{L}^{2}\left[k r, \delta_{J L}(k, r)\right],
$$

where $P_{L}$ is the uncoupled structure function, given by

$$
P_{L}\left[k r, \delta_{J L}(k, r)\right]=\cos \left[\delta_{J L}(k, r)\right] \hat{j}_{L}(k r)-\sin \left[\delta_{J L}(k, r)\right] \hat{n}_{L}(k r) .
$$

The integral expression for the variable phase is

$$
\delta_{J L}(k, r)=-\frac{1}{k} \int_{0}^{r} d r^{\prime} V_{J L}\left(r^{\prime}\right) P_{L}^{2}\left[k r^{\prime}, \delta_{J L}\left(k, r^{\prime}\right)\right] .
$$

For future use, we define the ratio $\rho_{J L}(k, r)$ as

$$
\rho_{J L}(k, r)=\frac{\delta_{J L}(k, r)}{\delta_{J L}(k, \infty)} .
$$

It represents the fraction of the phase shift that is generated by the part of the potential between the origin and the point $r$.

\section{RESULTS AND DISCUSSION}

The purpose of this work is to discuss the possibility of using $N \alpha$ scattering to study the tail of the two-pion exchange nucleon-nucleon potential. As stressed previously, the $\alpha$ particle is a spin-isospin scalar and hence it is very suited to the study of the intermediate-range part of the nuclear interaction. We therefore consider several realistic $N N$ potentials [1, 7, 9, 10, 12,20 and fold them into the $\alpha$ wave function, in order to obtain effective interactions, which are then used in the Schrödinger equation. The effective $N \alpha$ potential is dominated by scalar and isoscalar exchanges, giving rise to central and spin-orbit contributions, displayed in Fig. 11. In all cases, as expected, the spin-orbit component falls faster than the central one at large distances. Both components are negative there and hence, according to Eqs. (3.17) and (3.18), they interfere destructively when $J=L-\frac{1}{2}$ and constructively when $J=L+\frac{1}{2}$. This means that phase shifts for peripheral waves of $L$ are positive and larger for the latter class of waves.

We are interested in effects of the tail of the potential and hence it is important to establish, for each wave, a correlation between the energy and the region of the potential that determines the phase shifts. With that goal in mind, we have employed the variable phase method to evaluate the ratio $\rho_{J L}(k, r)$, defined in Eq. (4.9), for various energies, in order to determine the radii $R_{5}$ and $R_{10}$ for which $\rho_{J L}(k, r)$ is always less than 5 and $10 \%$, respectively. This means that $95 \%$ of the phase shift is generated in the region where $r>R_{5}$ and, similarly, $90 \%$ for $r>R_{10}$. Results for the Argonne potential [12] are presented in Table $\mathbb{H}$, where it is possible to notice some interesting features. The first of them is that, for a given value of $L$, the phases are slightly more sensitive to the short-range part of the potential for the channel with $J=L-\frac{1}{2}$. This feature of the problem can be fully appreciated in Fig. 2, that shows the ratios $\rho_{F_{5 / 2}}$ and $\rho_{F_{7 / 2}}$ at $15 \mathrm{MeV}$, which are representative of the other peripheral waves. The behavior of these curves are determined by the centrifugal barrier, responsible for the plateau close to the origin, combined with an effective potential which is repulsive at short distances and then attractive.

The $\alpha$ particle has a rms of about $1.6 \mathrm{fm}$ and, in a conservative approach, we may define the tail of the effective potential as beginning at $2.0 \mathrm{fm}$. Table then informs us that such a tail of the $N \alpha$ potential determines more than $95 \%$ of the phase shifts $\delta_{G_{7 / 2}}, \delta_{G_{9 / 2}}$ up to $50 \mathrm{MeV}$ and $\delta_{H_{9 / 2}}, \delta_{H_{11 / 2}}$ up to more than $80 \mathrm{MeV}$.

In Fig. 3 we display the phase shifts for waves with $2 \leq L \leq 5$. Inspecting the figures corresponding to the waves $F, G$, and $H$, one learns that the various potentials provide a coherent pattern, since all curves have the same general 
trends. The phases are sensitive to the tail of the effective potential and hence one could, in principle, discriminate the medium range features of the $N N$ interactions. In Fig. 3 we also include the available experimental values, taken from Refs. 23,26,25,24. The picture provided by these data, claimed to be precise, does not allow conclusions, for there are big discrepancies among phase shift analyses made by the various authors. So a drastic improvement in the precision of $N \alpha$ phase shifts is needed before they could be used to discriminate $N N$ potentials.

As a final comment, we would like to stress that our calculation is a very simple one and a more detailed study is required on the theoretical side. One of the points that deserves further attention concerns the construction of the $\alpha$ wave function, that should include $D$ components and, if possible, be calculated using realistic $N N$ potentials. Although it is reasonable to assume that intermediate states involving the ${ }^{3} \mathrm{Li}_{5},{ }^{2} \mathrm{He}_{5}$, or $\alpha$ excitations contribute little to peripheral scattering, it is also important to clarify this aspect of the problem. Finally, it would also be important to establish the quantitative role of the two-pion exchange three-body force, since its range is comparable to that of the TPEP. We are now investigating some of these questions and prefer to take the results of the present work as indicating reliably only that $N \alpha$ scattering allows the discrimination of the medium-range content of $N N$ potentials. In order to be able to select one or a class of potentials as being better than others one needs both more precise calculations and experimental phase shifts.

\section{ACKNOWLEDGMENT}

L. A. B. and R. H. were supported by Fundação de Amparo à Pesquisa do Estado de São Paulo (FAPESP).

[1] T. Hamada and J. D. Johnston, Nucl. Phys. 34, 382 (1962); T. Hamada, Y. Nakamura and R. Tamagaki, Progr. Theor. Phys. 33, 769 (1965); K. E. Lassila, M. H. Hull, H. M. Ruppel, F. A. MacDonald, and G. Breit, Phys. Rev. 126, 881 (1962); R. V. Reid, Ann. Phys. (N.Y.) 50, 411 (1968).

[2] G. Brown and D. W. Durso, Phys. Lett. 35B, 120 (1971).

[3] M. Chemtob, J. W. Durso, and D. O. Riska, Nucl. Phys. B38, 141 (1972).

[4] M. Lacombe, B. Loiseau, J. M. Richard, R. Vinh Mau, J. Coté, P. Pires, and R. de Tourreil, Phys. Rev. C 21, 861 (1980).

[5] M. H. Partovi and E. Lomon, Phys. Rev D 2, 1999 (1970).

[6] M. J. Zuilhof and J. A. Tjon, Phys. Rev. C 24, 736 (1981); 26, 1277 (1982).

[7] R. Machleidt, K. Holinde, and Ch. Elster, Phys. Lett. C149, 1 (1987).

[8] For a review see, R. Machleidt, Advances in Nuclear Physics, edited J. W. Negele and E. Vogt, (Plenum, New York, 1989), vol 19, p. 189.

[9] R. de Tourreil and D. W. L. Sprung, Nucl. Phys. A201, 193 (1973).

[10] R. de Tourreil, B. Rouben, and D. W. L. Sprung, Nucl. Phys. A242, 445 (1975).

[11] J. E. Lagaris and V. R. Pandharipande, Nucl. Phys. A359, 349 (1981).

[12] R. B. Wiringa, R. A. Smith, and T. L. Ainsworth, Phys. Rev. C 29, 1207 (1984).

[13] C. Ordóñez and U. van Kolck, Phys. Lett. 291B, 459 (1994).

[14] L. S. Celenza, A. Pantziris, and C. M. Shakin, Phys. Rev. C 46, 2213 (1992).

[15] J. L. Friar and S. A. Coon, Phys. Rev. C 49, 1272 (1994).

[16] C. A. da Rocha and M. R. Robilotta, Phys. Rev. C 49, 1818 (1994).

[17] C. Ordóñez, L. Ray, and U. van Kolck, Phys. Rev. Lett. 72, 1982 (1994).

[18] G. Höhler, H. P. Jacob, and R. Strauss, Nucl. Phys. B39, 237 (1972); R. Koch and E. Pietarinen, Nucl. Phys. A336, 331 (1980); G. Höhler, in Landolt-Börnstein Numerical Data and Functional Relationships in Science and Technology, edited by H. Schopper, Landolt-Börnstein, New Series, Group I, Vol. 9, Pt 2 (Springer Verlag, 1983).

[19] M. R. Robilotta, Nucl. Phys. A595, 171 (1995).

[20] M. R. Robilotta and C. A. da Rocha, Nucl. Phys. A615, 391 (1997).

[21] J-L. Ballot, M. R. Robilotta, and C. A. da Rocha, to appear in Phys. Rev. C.

[22] N. Kaiser, R. Brockmann, and W. Weise, Nucl. Phys. A625, 758 (1997).

[23] A. Houdayer, N. E. Davidson, S. A. Elbakr, A. M. Sourkes, W. T. H. van Oers, and A. D. Bacher, Phys. Rev. C 18, 1985 (1978).

[24] S. Burzynski, J. Campbell, M. Hammans, R. Henneck, W. Lorenzon, M.A. Pickar, and I. Sick, Phys. Rev. C 39, 56 (1989).

[25] H. Krupp, J. C. Hiebert, H. O. Klages, P. Doll, J. Hansmeyer, P. Plische, and J. Wilczynski, Phys. Rev. C 30, 1810 (1984).

[26] T. Saito, Nucl. Phys. A331, 477 (1979).

[27] A. C. C. Villari et al, Phys. Rev. Lett 71, 2551 (1993); M. S.Hussein, C. L. Lima, M. P. Pato, and C. A. Bertulani, ibid. 65, 839 (1990). 
[28] L. C. Chamon, D. Pereira, E. S. Rossi Jr, C. P. Silva, R. Lichtenthaler Filho, and L. C. Gomes, Nucl. Phys. A558, 305 (1995).

[29] H. Kanada, T. Kaneko, S. Nagata, and M. Nomoto, Progr. Theor. Phys. 61, 1327 (1979).

[30] F. Calogero, Nuovo Cimento 27, 261 (1963); Variable Phase Approach to Potential Scattering (Academic Press, New York, 1967). 


\begin{tabular}{|c|c|c|c|c|c|c|c|c|c|c|c|c|}
\hline \multirow[b]{2}{*}{$\underline{E}$} & \multicolumn{2}{|c|}{$F_{5 / 2}$} & \multicolumn{2}{|c|}{$F_{7 / 2}$} & \multicolumn{2}{|c|}{$G_{7 / 2}$} & \multicolumn{2}{|c|}{$G_{9 / 2}$} & \multicolumn{2}{|c|}{$H_{9 / 2}$} & \multicolumn{2}{|c|}{$H_{11 / 2}$} \\
\hline & $R_{5}$ & $R_{10}$ & $R_{5}$ & $R_{10}$ & $R_{5}$ & $R_{10}$ & $R_{5}$ & $R_{10}$ & $R_{5}$ & $R_{10}$ & $R_{5}$ & $R_{10}$ \\
\hline 5 & 2.12 & 3.26 & 2.96 & 3.10 & 3.24 & 3.48 & 3.10 & 3.34 & 3.62 & 3.96 & 3.50 & 3.86 \\
\hline 10 & 1.96 & 3.22 & 2.24 & 3.06 & 3.18 & 3.38 & 3.04 & 3.24 & 3.48 & 3.78 & 3.36 & 3.68 \\
\hline 15 & 1.84 & 2.22 & 2.00 & 3.04 & 3.14 & 3.32 & 2.98 & 3.18 & 3.38 & 3.66 & 3.26 & 3.54 \\
\hline 20 & 1.74 & 2.02 & 1.86 & 3.02 & 3.10 & 3.26 & 2.94 & 3.12 & 3.30 & 3.56 & 3.18 & 3.44 \\
\hline 30 & 1.56 & 1.78 & 1.68 & 1.98 & 3.06 & 3.20 & 2.88 & 3.02 & 3.20 & 3.40 & 3.06 & 3.28 \\
\hline 40 & 1.40 & 1.58 & 1.52 & 1.74 & 2.14 & 3.14 & 2.82 & 2.96 & 3.12 & 3.30 & 2.96 & 3.16 \\
\hline 50 & 1.26 & 1.40 & 2.23 & 2.95 & 1.94 & 2.34 & 2.80 & 2.92 & 3.06 & 3.22 & 2.90 & 3.08 \\
\hline 60 & 1.08 & 1.20 & 1.97 & 2.91 & 1.80 & 2.06 & 2.00 & 2.88 & 3.02 & 3.16 & 2.84 & 3.00 \\
\hline 70 & 0.78 & 0.86 & 1.12 & 1.26 & 1.68 & 1.88 & 1.84 & 2.86 & 3.00 & 3.12 & 2.80 & 2.94 \\
\hline 80 & 0.86 & 1.06 & 0.98 & 1.08 & 1.56 & 1.74 & 1.72 & 1.98 & 2.26 & 3.08 & 2.76 & 2.90 \\
\hline
\end{tabular}

TABLE I. Radii $R_{5}$ and $R_{10}$, in fm, as function of the energy $\mathrm{E}$, in $\mathrm{MeV}$, for which the normalized phase shift, calculated for the Argonne potential, is always less than 5\% and 10\%, respectively. It is worth noting the non monotonic behavior of the $F_{5 / 2}$ wave, which is due to a change in sign in the Argonne phase shift just before $50 \mathrm{MeV}$. 
FIG. 1. Effective $N \alpha$ central (continuous line) and spin-orbit (dashed line) potentials for the Paris 顿, Bonn (OBEPR) 牙, supersoft core C [9], dTRS [10], Argonne [12], and chiral [20] interactions.

FIG. 2. Phase shifts normalized to 1 [Eq. 4.9 at $15 \mathrm{MeV}$, as function of $r$ for the waves $F_{5 / 2}$ (dashed line) and $F_{7 / 2}$ (continuous line).

FIG. 3. $N \alpha$ phase shifts for the Paris (P), Bonn (B), supersoft core C (S), dTRS (T), Argonne (A), and chiral (C) $N N$ interactions. The experimental results are taken from Refs. [23] $(\circ), 24(\diamond), 25](+)$, and 26$](\times)$. 

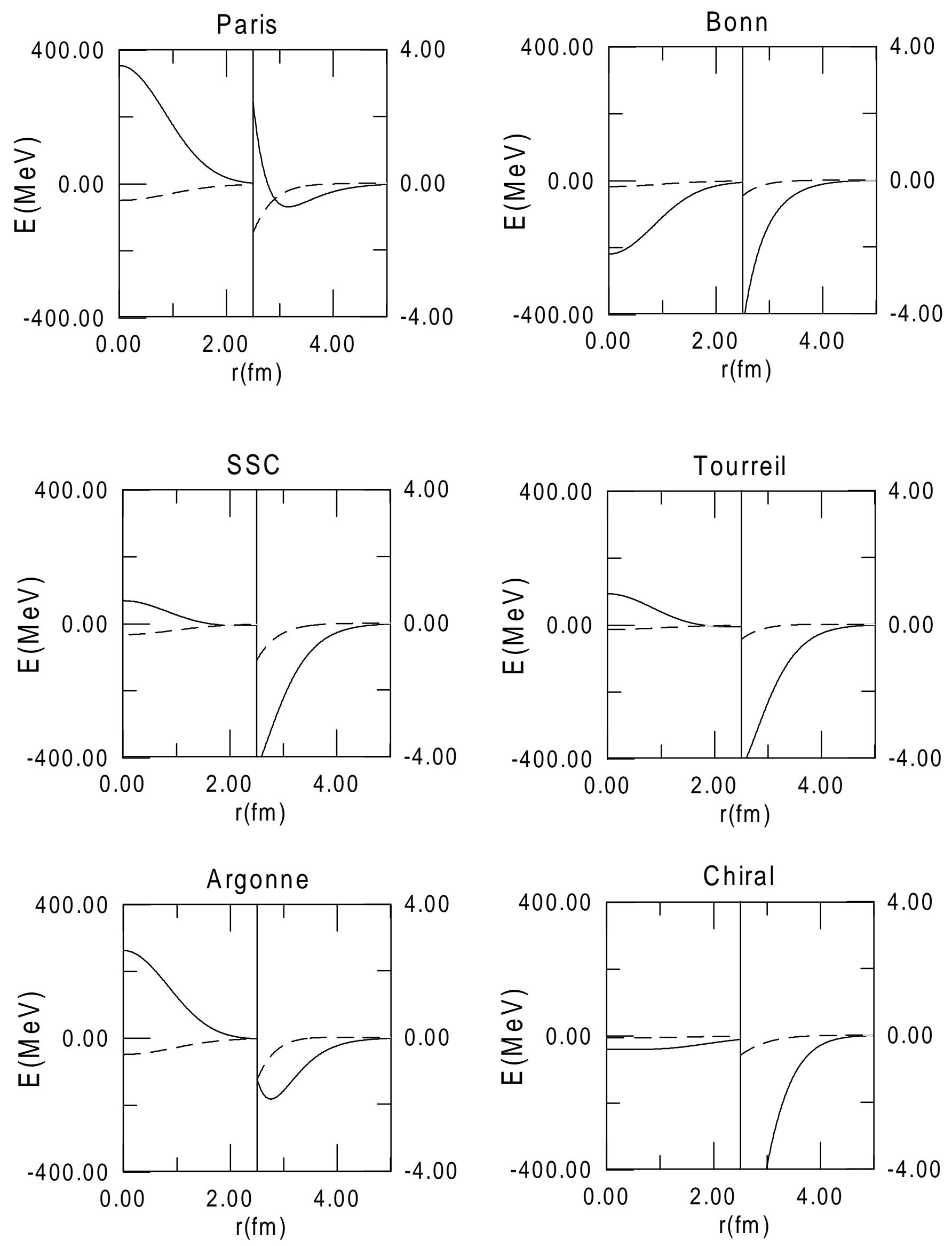

Fig. 1 


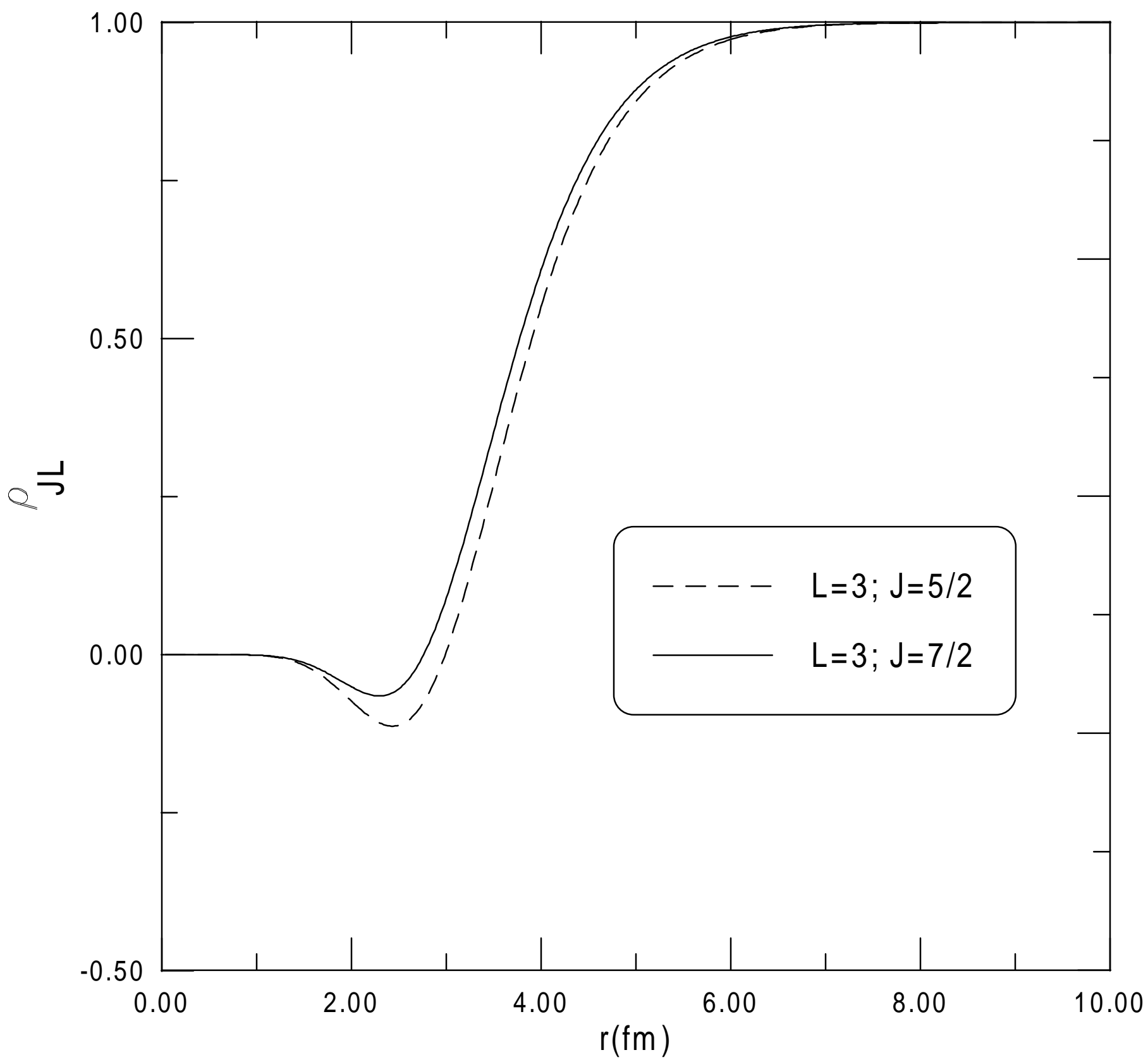

Fig. 2 
Fig. $3 a-3 b$
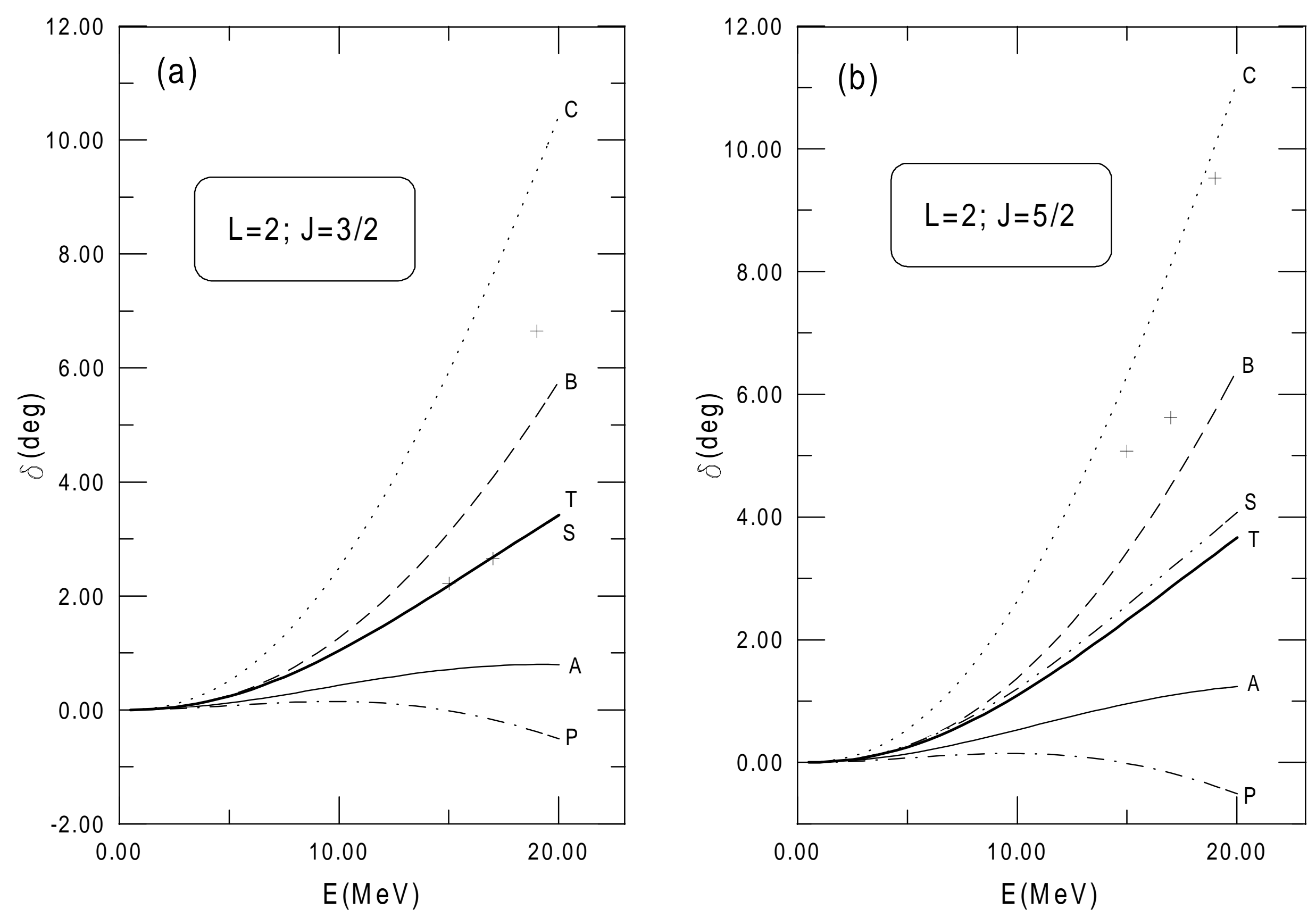
Fig. $3 c-3 d$
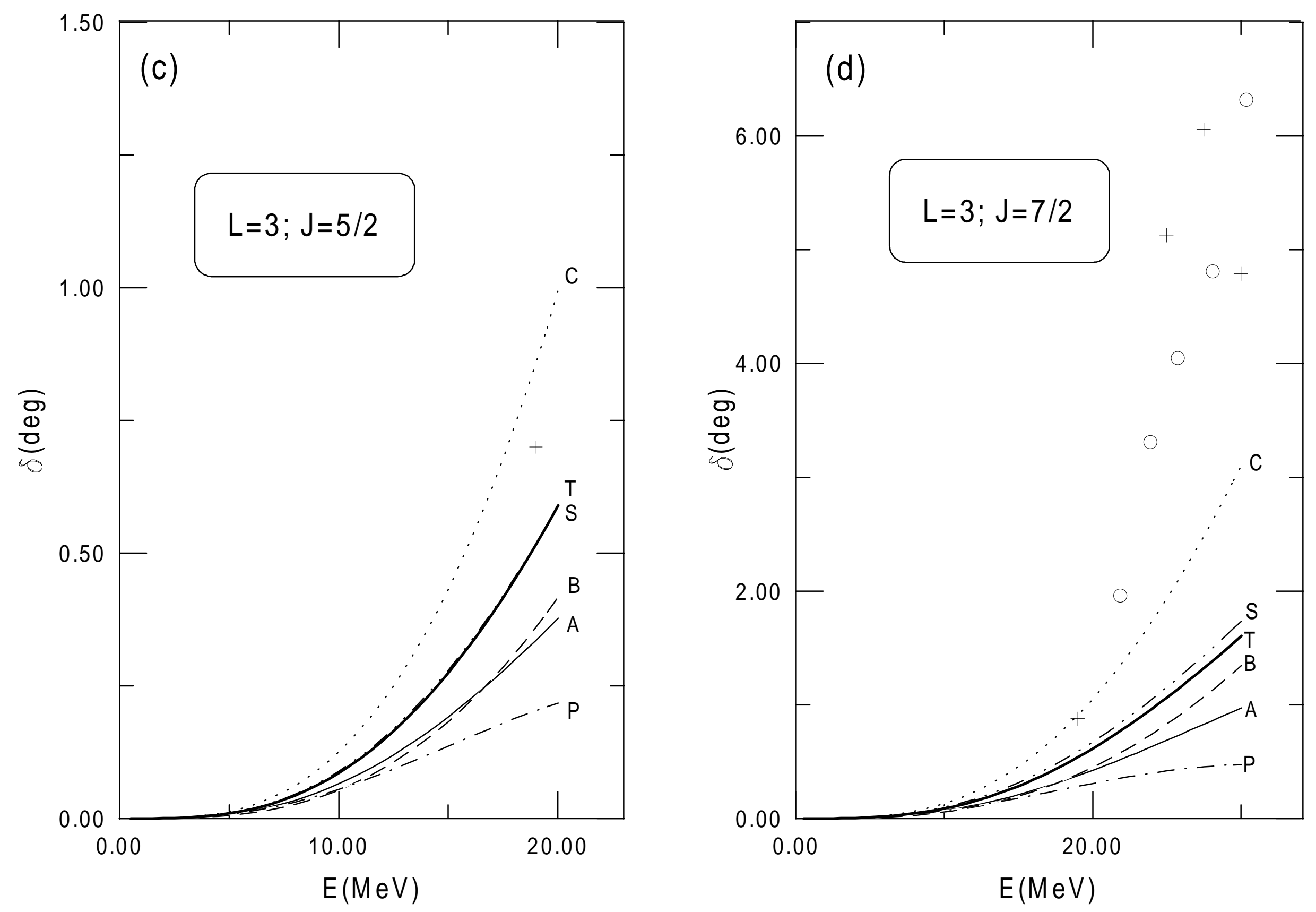
Fig. $3 e-3 f$
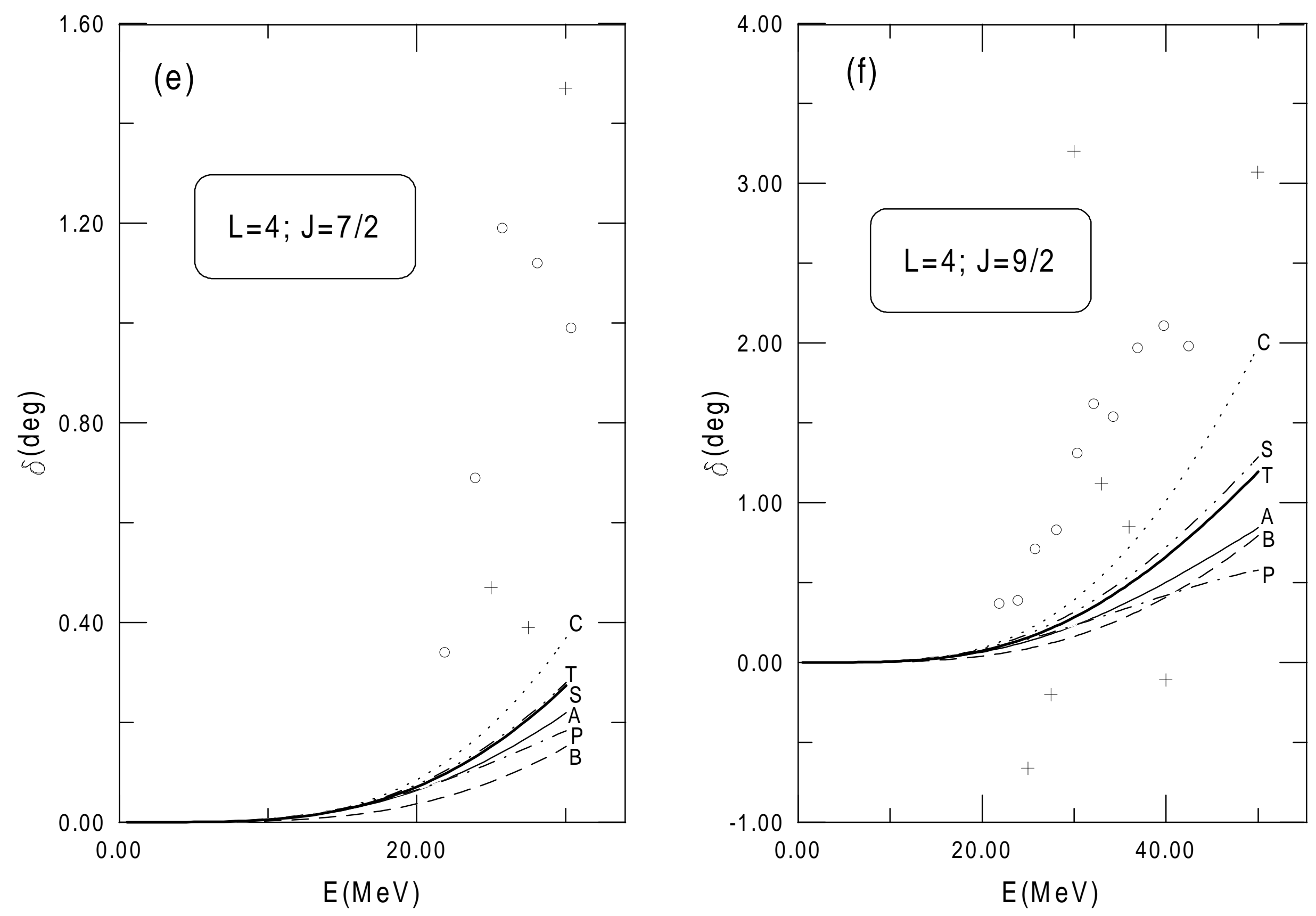
Fig. $3 g-3 h$
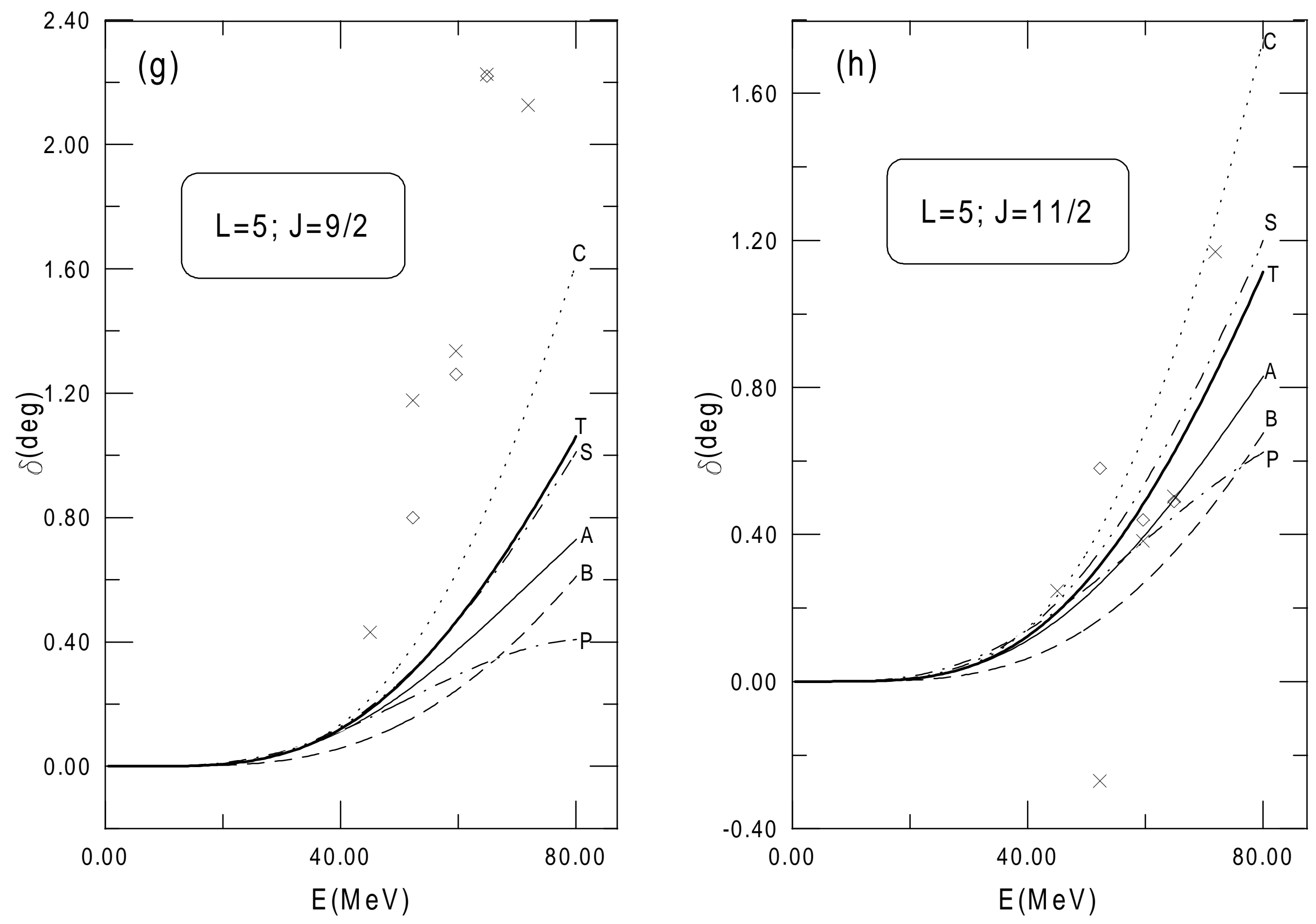